\title{
Migration of Peripherally Inserted Central Catheter after Contrast-Enhanced Computed Tomography
}

\author{
Jonghoo Lee and Gil Myeong Seong* \\ Department of Internal Medicine, Jeju National University School of Medicine, Jeju, Korea
}

A 35-year-old man with end-stage renal disease was admitted for chronic respiratory failure. During hospitalization, arteriovenous graft malfunction occurred and a computed tomography (CT) angiography was planned. A 5 French power injectable peripherally inserted central catheter (PICC, Turbo-Ject ${ }^{\circledR}$; Cook Medical, Bloomington, IN) was inserted under fluoroscopic guidance, and located in the distal superior vena cava and fixed to the skin with sutures (Fig. 1A). Surprisingly, two days after catheter insertion, chest X-rays showed the catheter had become displaced into the ipsilateral internal jugular vein (Fig. 1B).
The insertion site was clear, and no evidence of other external force was applied. During the review of CT performed one day ago, we found that the position of the catheter changed after injection of the contrast agent (Fig. 1C, D). An upper extremity CT angiography was performed with $150 \mathrm{~mL}$ of iodinated contrast infused at a rate of $5 \mathrm{~mL} / \mathrm{s}$. There was no problem with contrast injection, but the position of the catheter was changed in the delayed image performed three minutes later. The catheter was removed after positional changes were noted and there were no complications. This case highlights that displacement of
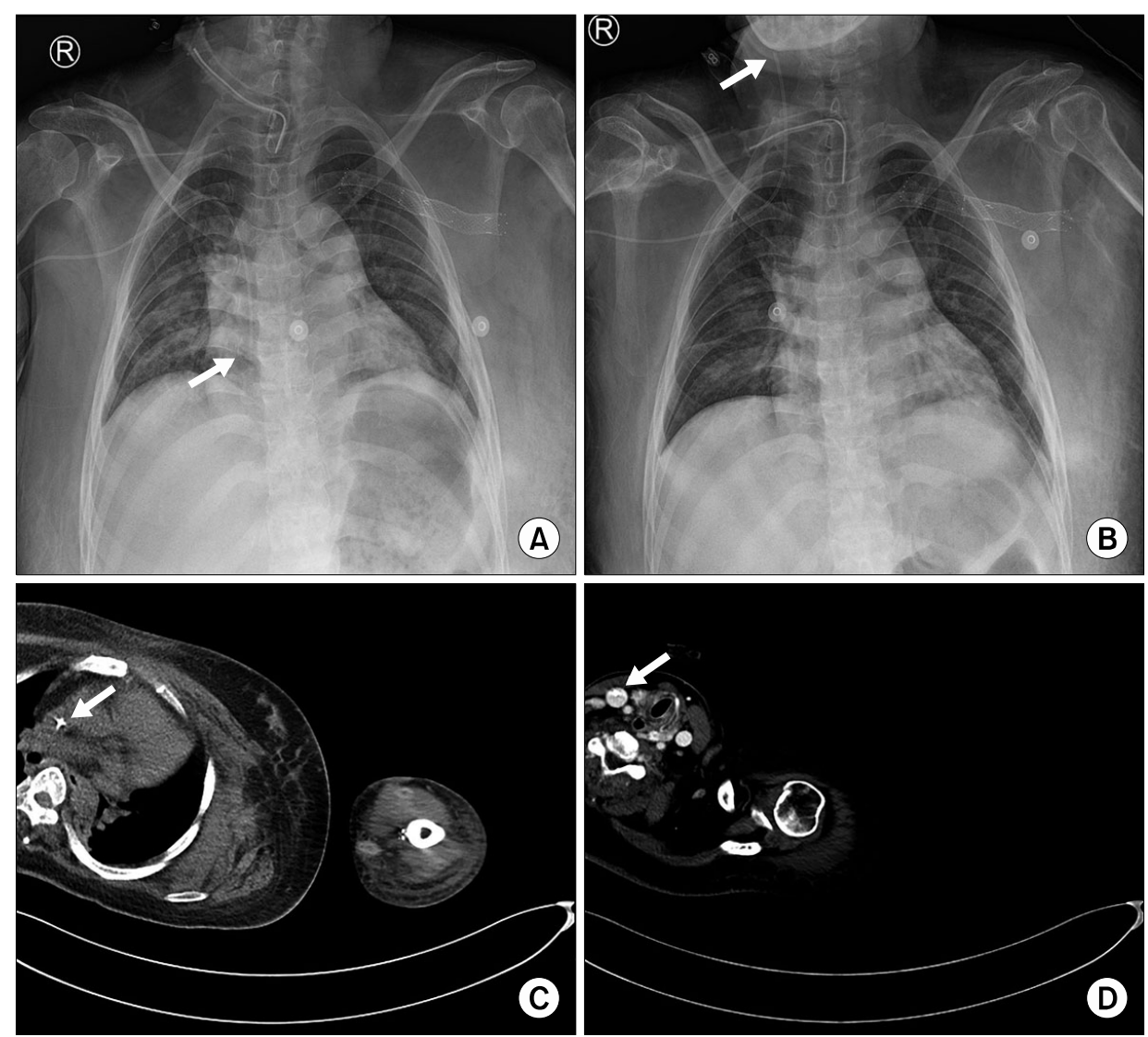

Fig. 1. Migration of peripherally inserted central catheter (arrows). (A, C) Initial placement of the right sided peripherally inserted central catheter in the distal superior vena cava; and (B, D) migration of the line to the right internal jugular vein.

Corresponding Author:

Gil Myeong Seong

Department of Internal Medicine, Jeju National University School of Medicine, 15 Aran 13-gil, Jeju 63241, Korea Tel: +82-64-717-1601, Fax: +82-64-717-1131, E-mail: rolland0211@gmail.com
Article History:

Received March 13, 2019

Revised April 6, 2019

Accepted April 10, 2019 
power injectable PICC can occur even within the authorized infusion rate and reminds us again that it is essential to compare previous chest $\mathrm{x}$-rays for detection of complications associated with indwelling devices.

\section{CONFLICT OF INTEREST STATEMENT}

None declared.

This is an Open Access article distributed under the terms of the Creative Commons Attribution Non-Commercial License (http://creativecommons.org/licenses/ by-nc/4.0) which permits unrestricted non-commercial use, distribution, and reproduction in any medium, provided the original work is properly cited. 